\title{
The phonon bottleneck in paramagnetic crystals
}

\author{
A. M. STONEHAM \\ Theoretical Physics Division, Atomic Energy Research Establishment, Harwell, \\ Didcot, Berks.
}

MS. received 19th January 1965, in revised form 14th July 1965

\begin{abstract}
A unified treatment of phonon bottleneck theory is given, using a thermodynamic method which includes both the direct and Orbach processes. The key parameter is the phonon relaxation time, which is calculated in a variety of cases, including several in which it is determined by magnetic impurities. The theory is extended to include arbitrary spin resonance line shapes and cases where the phonon relaxation time is energy dependent.
\end{abstract}

\section{Introduction}

Spin-lattice relaxation rates are usually calculated for a single ion relaxing to a phonon system which is not appreciably perturbed by the interaction. Although this is often valid, it is not a good model when the rate-determining process is some process in the phonon system rather than the transfer of energy from the spin to the lattice. This situation is known as the 'phonon bottleneck' (Van Vleck 1951 a) and the theory of this effect is the subject of this paper.

The model we use is a thermodynamic model, which has been used in related contexts by Casimir (1939) and by Gorter, Van der Marel and Bolger (1955). It assumes that the complete experimental arrangement may be thought of as consisting of three partsthe spins, the band of phonons which are responsible for spin-lattice relaxation (the

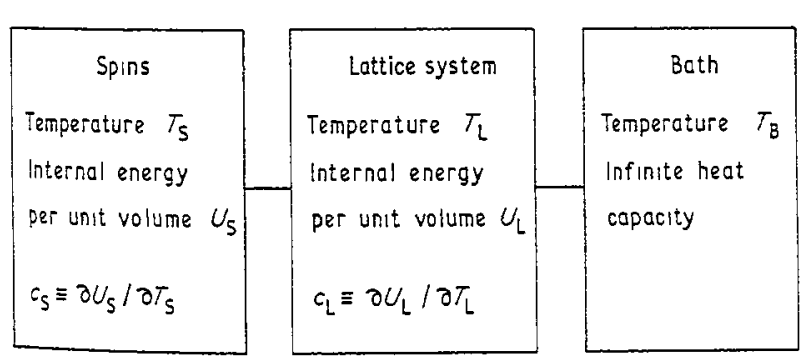

Figure 1.

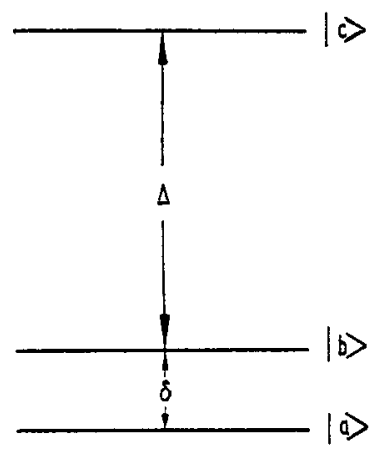

Figure 2. Energy level diagram for the spin species discussed; $\Delta \gg k T \gg \delta$.

lattice system) and a bath of supposedly infinite heat capacity-and that each of these three parts may be described by a temperature. This arrangement is shown in figure 1. The equations which describe the transfer of energy between these parts are then written down and solved. We shall also assume that we may linearize our equations by neglecting 
high powers of temperature differences between the three parts. This is a valid assumption, as most measurements of relaxation times are made when the temperature differences are about $5 \%$ or less of the bath temperature.

To be specific, we consider a system of spins whose energy levels are shown in figure 2; this is the simplest system that shows all the points of interest. The numbers of spins per unit volume in states $|\mathrm{a}\rangle$ and $|\mathrm{b}\rangle$ are $N_{\mathrm{a}}$ and $N_{\mathrm{b}}$ respectively; the energy separation of these states may be caused by an applied magnetic field or by a crystal field of low symmetry. We are interested in the recovery of $N_{\mathrm{a}}$ and $N_{\mathrm{b}}$ when their populations have been disturbed. The spin temperature $T_{\mathrm{s}}$ is defined by

$$
N_{\mathrm{a}}=N_{\mathrm{b}} \exp \left(\delta / k T_{\mathrm{s}}\right) \text {. }
$$

We shall assume $\Delta \gg k T \gg \delta$ throughout this article, as this simplifies many formulae and retains all the physically important features. Thus

$$
T_{\mathrm{S}}=\frac{\delta}{2 k} \frac{N_{\mathrm{a}}+N_{\mathrm{b}}}{N_{\mathrm{a}}-N_{\mathrm{b}}}
$$

When phonons of a single energy are responsible for spin-lattice relaxation, the lattice temperature $T_{\mathrm{L}}$ is defined in terms of their Einstein-Bose occupation number

$$
n(E)=\left\{\exp \left(\frac{E}{k T_{\mathrm{I}}}\right)-1\right\}^{-1}
$$

Thus, for $E=\delta$,

$$
T_{\mathrm{L}}=\frac{\delta}{k} n(\delta)
$$

Experimentally, one measures the rate at which $T_{\mathfrak{S}}$ returns to $T_{\mathrm{B}}$ after the spin system has been disturbed. The observed relaxation time, defined by

$$
\dot{T}_{\mathrm{S}}=-\frac{1}{\tau_{\mathrm{obs}}}\left(T_{\mathrm{S}}-T_{\mathrm{B}}\right)
$$

depends on both the rate at which energy is transferred from the spins to the lattice system and on the rate at which the lattice system transfers energy to the bath. These two rates are described, respectively, by the spin-lattice relaxation time $\tau_{0}$ defined by

$$
\dot{T}_{\mathrm{S}}=-\frac{1}{\tau_{0}}\left(T_{\mathrm{S}}-T_{\mathrm{L}}\right)
$$

and the phonon relaxation time $\tau_{\mathrm{ph}}$ defined by

$$
\dot{T}_{\mathrm{L}}=-\frac{1}{\tau_{\mathrm{ph}}}\left(T_{\mathrm{L}}-T_{\mathrm{B}}\right)
$$

In the equation defining $\tau_{\mathrm{ph}}$, terms from interaction with the spins have been omitted. This definition of $\tau_{\mathrm{ph}}$ is equivalent, if the limit $\Delta \gg k T \gg \delta$ is assumed, to that used by Faughnan and Strandberg (1961):

$$
\dot{n}(E)=-\frac{1}{\tau_{\mathrm{ph}}}\left\{n(E)-n_{0}(E)\right\}
$$

in which the occupation numbers $n(E), n_{0}(E)$ are to be evaluated at $T_{\mathrm{L}}, T_{\mathrm{B}}$ respectively. 
In $\S 2$ we examine the dependence of $\tau_{\text {obs }}$ on $\tau_{0}$ and $\tau_{\mathrm{ph}}$ for the so-called direct and Orbach spin-lattice relaxation processes. Some of the results have been given previously using the alternative rate equation approach (e.g. Faughnan and Strandberg 1961). We reformulate the theory in terms of the thermodynamic approach, and derive the results needed in subsequent sections. The advantages of the thermodynamic approach are that it is relatively simple, so one can derive general results and analyse the accuracy of the approximations involved. It can be extended to complicated situations without formal difficulty. Faughnan and Strandberg write down coupled rate equations for the numbers of spins and phonons resonant with the spins. This is particularly convenient when the linear approximation is invalid, although in practice the equations are nearly always linearized before being solved (e.g. Scott and Jeffries 1962). To the linear approximation the two approaches give the same results, although there has been no detailed comparison of the circumstances in which the two methods are valid. The calculations of $\S 2$ are simplified by assuming that the spin-phonon interaction is constant inside some band width and zero outside it. This approximation has been used in all previous treatments and is relaxed in $\S 3$, in which we take into account the variation of the spin-phonon interaction and the phonon relaxation time with energy. In $\S 4$ we calculate phonon relaxation times explicitly, including several mechanisms involving impurities which have not been discussed previously. One of these mechanisms may be responsible for phonon relaxation in crystals of MgO doped with $\mathrm{Co}^{2+}$ (Whittlestone 1964, Ph.D. Thesis, University of Bristol). Comparison with experiment in $\S 5$ shows that it is possible to find explanations consistent with observation in many cases, although it is difficult to draw firm conclusions as important factors, such as the condition of the crystal surface and trace impurity concentrations, are hard to measure. Several distinct phonon relaxation mechanisms lead to the $T^{\sim 2}$ dependence of the direct process relaxation time, which is usually regarded as a symptom of a bottleneck. Two of the mechanisms discussed in $\$ 4$ (the 'anharmonic' and 'one-phonon' processes) lead to a different temperature dependence.

\section{The thermodynamic approach to the phonon bottleneck}

The linearized equations describing the transfer of energy between the systems shown in figure 1 may be written

$$
\begin{aligned}
& \frac{d U_{\mathrm{S}}}{d t}=c_{\mathrm{S}} \frac{d T_{\mathrm{S}}}{d t}=\alpha\left(T_{\mathrm{L}}-T_{\mathrm{S}}\right) \\
& \frac{d U_{\mathrm{L}}}{d t}=c_{\mathrm{L}} \frac{d T_{\mathrm{L}}}{d t}=\alpha\left(T_{\mathrm{S}}-T_{\mathrm{L}}\right)+\beta\left(T_{\mathrm{B}}-T_{\mathrm{L}}\right) .
\end{aligned}
$$

The coefficients are related to $\tau_{0}$ and $\tau_{\mathrm{ph}}$, defined in $\S 1$, by $\tau_{0}=c_{\mathrm{S}} / \alpha$ and $\tau_{\mathrm{ph}}=c_{\mathrm{L}} / \beta$. $\alpha$ and $\beta$ depend on $T_{\mathrm{L}}$ and $T_{\mathrm{S}}$ for a given $T_{\mathrm{B}}$, for example

$$
\alpha=\alpha\left(T_{\mathrm{B}}\right)+\text { corrections of order } \frac{T_{\mathrm{S}}-T_{\mathrm{B}}}{T_{\mathrm{B}}}, \frac{T_{\mathrm{L}}-T_{\mathrm{B}}}{T_{\mathrm{B}}} .
$$

These corrections to $\alpha$ and $\beta$ lead to terms in (1) and (2) which are quadratic in $T_{\mathrm{S}}-T_{\mathrm{B}}$ and $T_{\mathrm{L}}-T_{\mathrm{B}}$ and are dropped in linearizing these equations.

The term $d U_{L} / d t$ in (2) is typically $10^{-5}$ of the others. If we neglect this term, assuming that the net rate at which the lattice system gains energy is insignificant, 
we find, independent of the details of the relaxation processes,

$$
\begin{aligned}
\tau_{\text {obs }} & =\frac{c_{\mathrm{S}}}{\alpha}\left(1+\frac{\alpha}{\beta}\right) \\
& =\tau_{0}+\tau_{\mathrm{ph}} \frac{c_{\mathrm{S}}}{c_{\mathrm{L}}} .
\end{aligned}
$$

If we had retained $d U_{\mathrm{I}} / d t$ the recovery of $T_{\mathrm{S}}$ would be described by two time constants. One, equivalent to $\tau_{\mathrm{obs}}$, describes the mutual progress of $T_{\mathrm{S}}$ and $T_{\mathrm{L}}$ to $T_{\mathrm{B}}$. The other corresponds to the tendency of $T_{\mathrm{S}}$ and $T_{\mathrm{L}}$ to equalize; it is very short, vanishing with $d U_{\mathrm{L}} / d t$, and is therefore very difficult to detect.

From (3) we see that unless the term in $\tau_{\mathrm{ph}}$ is small, the rate at which the spin system loses energy is limited by the transfer of energy between the lattice and the bath systems. Another feature is that $T_{\mathrm{L}}$ is raised from its equilibrium value $T_{\mathrm{B}}$ to

$$
T_{\mathrm{L}}=T_{\mathrm{B}}+\frac{\alpha / \beta}{1+\alpha / \beta}\left(T_{\mathrm{S}}-T_{\mathrm{B}}\right)
$$

so that $T_{\mathrm{L}}$ is close to $T_{\mathrm{S}}$ when $\alpha$ greatly exceeds $\beta$.

The specific heat per unit volume $c_{\mathbb{S}}$ of the spin system described in $\S 1$ is

$$
\begin{aligned}
c_{\mathrm{S}} & =\frac{\partial U_{\mathrm{S}}}{\partial T_{\mathrm{S}}}=-\frac{\partial}{\partial T_{\mathrm{S}}}\left\{\frac{1}{2} \delta\left(N_{\mathrm{a}}-N_{\mathrm{b}}\right)\right\} \\
& \simeq \frac{1}{4} N\left(\frac{\delta}{k T_{\mathrm{S}}}\right)^{2} k
\end{aligned}
$$

where $N=N_{\mathrm{a}}+N_{\mathrm{b}}$. For $\delta=0.3 \mathrm{~cm}^{-1}, T_{\mathrm{S}}=4.2^{\circ} \mathrm{K}$ and $N=3 \times 10^{18} \mathrm{ions} / \mathrm{cm}^{3}$ this is about $1 \mathrm{erg} \mathrm{cm}^{-3} \operatorname{degk}^{-1}$.

The long wave approximation for the phonon system will be used in calculating $c_{\perp}$. The number of modes whose energy lies within $d E$ of $E$ is thus

$$
\rho(E) d E=\frac{3}{2 \pi^{2} \hbar^{3}} \frac{1}{v^{3}} E^{2} d E .
$$

where $v$ is an average $\left(v_{1}^{-3}+2 v_{t}^{-3}\right)^{-1 / 3}$ of the velocities $v_{1}$ and $v_{t}$ of longitudinal and transverse sound waves.

To go further, the several spin-lattice relaxation processes must be treated separately. If the relative populations of $|a\rangle$ and $|b\rangle$ are altered, the equilibrium distribution may be restored by one of three principal mechanisms (Orbach 1961):

(i) The direct process. In this process the spin transitions from $|b\rangle$ to $|a\rangle$ occur with the emission of a single phonon with energy close to $\delta$. For the moment we assume that the phonons with energy between $\delta$ and $\delta+\Gamma$ constitute the lattice system and interact equally strongly with the spins, whereas phonons with other energies do not interact with the spins at all. This simplifying assumption will be relaxed in $\S 3$.

The lattice specific heat is

$$
\begin{aligned}
c_{\mathrm{L}} & \left.=\frac{\partial U_{\mathrm{L}}}{\partial T_{\mathrm{L}}}=\frac{\partial}{\partial T_{\mathrm{L}}}\left[\Gamma \rho(\delta)\left\{n(\delta)+\frac{1}{2}\right\}\right]\right] \\
& \simeq \rho(\delta) \Gamma k
\end{aligned}
$$

which is typically of the order of $10^{-3} \mathrm{erg} \mathrm{cm}^{-3} \mathrm{degk}^{-1}$, so $c_{\mathrm{S}} / c_{\mathrm{L}}$ is typically $10^{3}$. It 
then follows from (3), (4) and (6) that

$$
\tau_{\text {obs }}=\tau_{0}+\tau_{\mathrm{ph}} \frac{N}{\rho(\delta) \Gamma}\left(\frac{\delta}{2 k T}\right)^{2} .
$$

The coefficient of $\tau_{\mathrm{ph}}$ is independent of $\delta$, and therefore independent of an applied magnetic field, as $\rho(\delta)$ is proportional to $\delta^{2}$. If $\Gamma$ is proportional to the spin concentration, $N / \Gamma$ is independent of this concentration; in this case the coefficient of $\tau_{p h}$ is a function of temperature only, and

$$
\tau_{\text {obs }}=A T^{-1}+D T^{-2}
$$

as $\tau_{0}$ is proportional to $T^{-1}$ for the direct process. $D$ has the temperature, field and concentration dependence of $\tau_{\mathrm{ph}}$.

(ii) The Raman process. In this process the spin absorbs a phonon of one energy and emits a phonon of a different energy. This is a non-resonant process in which all the phonons of the crystal can participate. $c_{\mathrm{L}}{ }^{R}$ is of the order of the total lattice specific heat, and cubic in $T_{\mathrm{L}}$ in the Debye approximation. As this is typically three orders of magnitude larger than $c_{\mathbb{S}}$ there is little prospect of a bottleneck. This process will not be discussed further.

(iii) The Orbach process. In going from state $|\mathrm{b}\rangle$ to $|a\rangle$ the spin makes a real transition from $|b\rangle$ to an excited state $|c\rangle$, absorbing a phonon of energy $\Delta$, and subsequently emits a phonon of energy $\Delta+\delta$ in going to $|a\rangle$. This is a resonant process in which the spins interact with two bands of phonons-those with energy close to $\Delta$ and those with energy close to $\Delta+\delta$-and these comprise the lattice system. The definition of $T_{\mathrm{L}}$ in $\S 1$ is no longer adequate, as two temperatures are necessary to give the phonon occupation numbers for the two bands. The number of phonons $\Pi$ in the lattice system is unaltered by the Orbach process

$$
\Pi=\rho(\Delta) \Gamma_{0}\{n(\Delta)+n(\Delta+\delta)\}
$$

in which the occupation numbers can be calculated using the bath temperature $T_{\mathrm{B}}$. $T_{\mathrm{B}}$ gives the total number of phonons in the two bands which form the lattice system, whereas the lattice temperature $T_{\mathrm{L}}$ describes the relative populations of these bands. The distribution law for the phonons of energy $\Delta$ and $\Delta+\delta$ has the form

$$
n(E)=\left\{\frac{1}{\lambda} \exp \left(\frac{E}{k T_{\mathrm{L}}}\right)-1\right\}^{-1}
$$

(Tolman 1938, $\S 91$ ), in which $\lambda$ is fixed by the condition that the total number of these phonons is $\Pi$, independent of $T_{\mathrm{L}}$. Thus the relative occupancy of the two bands is measured by

$$
T_{\mathrm{L}}=\frac{\delta}{2 k} \frac{n(\Delta)+n(\Delta+\delta)}{n(\Delta)-n(\Delta+\delta)}
$$

The rate equations for the Orbach process confirm that the spins tend to a distribution with $T_{\mathrm{S}}=T_{\mathrm{L}}$. If there are more phonons of energy $\Delta+\delta$ than of energy $\Delta, T_{\mathrm{L}}$ will be negative; $n(\Delta+\delta)$ may exceed $n(\Delta)$ as these are the occupation numbers of different phonon modes. The lattice temperature for the direct process, $\delta n(\delta) / k$, cannot be negative as this would imply a negative occupation number $n(\delta)$.

The internal energy per unit volume $U_{\mathrm{L}}{ }^{0}$ is, to order $\delta / \Delta$,

$$
\begin{aligned}
U_{\mathrm{L}}{ }^{0} & =\rho(\Delta) \Gamma_{0}\{\Delta n(\Delta)+(\Delta+\delta) n(\Delta+\delta)\}+\text { zero-point energy } \\
& =\left(\Delta+\frac{1}{2} \delta\right) \Pi+\text { zero-point energy }-\Pi \frac{\delta^{2}}{4 k T_{\mathrm{I}}}
\end{aligned}
$$


so that

$$
c_{\mathrm{L}}^{0}=\rho(\Delta) \Gamma_{0} \exp \left(\frac{-\Delta}{k T_{\mathrm{B}}}\right)\left(\frac{\delta}{k T_{\mathrm{L}}}\right)^{2} k
$$

The observed relaxation time is

$$
\tau_{\text {obs }}{ }^{0}=\tau_{0}{ }^{0}+\tau_{\text {ph }}{ }^{0} \frac{N \exp (\Delta / k T)}{2 \rho(\Delta) \Gamma_{0}} .
$$

If there is an excess of ions in state $|b\rangle$ over the equilibrium value, phonons of energy $\Delta$ are destroyed and those of energy $\Delta+\delta$ created. The lattice temperature $T_{\mathrm{L}}$ may thus be raised appreciably, and (9) shows the effect on the relaxation rate.

\subsection{Relation to the theory of thermal conductivity}

Equations (7) and (9) may be rewritten in terms of the phonon relaxation time $\tau_{0}(E)$ of thermal conductivity theory (Klemens 1958). In general, $\tau_{c}$, which describes the relaxation of phonons with a given wave vector, is not simply related to $\tau_{\text {ph }}$, which describes the relaxation of phonons with a given energy. For a phonon mode resonant with the spins the relaxation corresponding to $\tau_{\mathrm{o}}$ is usually dominated by the elastic scatter by the spins of phonons from one resonant mode to another. If we neglect other mechanisms, then for direct process phonons, of energy $\delta$,

$$
\tau_{0}(\delta)=\frac{\rho(\delta) \Gamma \tau_{0}}{N}\left(\frac{2 k T}{\delta}\right)^{2}
$$

following Orbach (1962); for the phonons of energy $\Delta$ involved in the Orbach process

$$
\tau_{c}(\Delta)=2 \frac{\rho(\Delta) \Gamma_{0} \tau_{0}{ }^{0}}{N} \exp \left(-\frac{\Delta}{k T}\right) .
$$

Equations (7) and (9) become

and

$$
\tau_{\text {obs }}=\tau_{0}\left\{1+\frac{\tau_{\mathrm{ph}}(\delta)}{\tau_{\mathrm{c}}(\delta)}\right\}
$$

$$
\tau_{\text {obs }}^{0}=\tau_{0}^{0}\left\{1+\frac{\tau_{\mathrm{ph}}(\Delta)}{\tau_{\mathrm{c}}(\Delta)}\right\}
$$

\section{The effect of line shape}

In the last section we assumed that the spin-phonon interaction was constant within a band of width $\Gamma$ and zero outside it, and that the phonon relaxation time was independent of the phonon energy. These assumptions have been made in all previous treatments of this problem. The theory is generalized in this section.

When there is no bottleneck the relaxation rate is the sum of the contributions of phonons of different energies:

$$
\frac{1}{\tau_{0}}=\int d E \frac{1}{\tau_{0}(E)}
$$

Here the contribution of phonons in the infinitesimal band $E$ to $E+d E$ is $d E / \tau_{0}(E)$; 
$\tau_{0}(E)$ includes the variation with $E$ of the spin-phonon interaction and of the number of spins with resonance energy $E$. Standard spin-lattice relaxation theory (Orbach 1961) shows that

$$
\frac{1}{\tau_{0}(E)} \sim E \rho(E) g(E)
$$

where $g(E)$ is the spin resonance line shape for a microwave phonon experiment. As $E \rho(E)$ usually varies little when $g(E)$ is appreciable we write

$$
\frac{1}{\tau_{0}(E)} \simeq \frac{g(E)}{\tau_{0}}
$$

For spins with effective spin $S^{\prime}=\frac{1}{2}, g(E)$ is the same as the line shape measured in 'ordinary' spin resonance experiments (Stoneham 1965), and when $S^{\prime}>\frac{1}{2}$ the two shapes may be very similar (Loudon 1960). The variation of the phonon relaxation time with energy is written

$$
\frac{1}{\tau_{\mathrm{ph}}(E)}=\frac{\gamma(E)}{\tau_{\mathrm{ph}}}
$$

A new feature becomes important when there is a bottleneck, for there may be direct transfer of energy between phonons resonant with spins having different resonant frequencies or between the spins themselves. There are two extreme approximations to the rate of this spectral diffusion of energy. On the one hand it can be assumed to be so fast that $T_{\mathrm{S}}$ is independent of the spin resonance frequency and that $T_{\mathrm{L}}$ is independent of the resonant phonon energy. In this case $\alpha$ and $\beta$ of (3) are each replaced by their integrals over energy, which are also weighted by the appropriate line shapes. On the other hand we can assume that spectral diffusion is so slow that the spin-phonon systems within each infinitesimal bandwidth $d E$ relax independently of those in similar bands. Then, in general, the $T_{\mathrm{s}}$ for bands of different energy will differ, and will equalize only by the interaction of the spins with their resonant phonons, and of the phonons with the bath. Similarly $T_{\mathrm{L}}$ will vary from band to band. We adopt this assumption, assuming that we may neglect spectral diffusion in calculations of the observed relaxation rate. There is evidence that energy diffusion of the type of interest is slow in the phonon system (Brya and Wagner 1965); Portis (1956) and Gill and Meredith (1965) have discussed spectral diffusion in the spin system, which should certainly be slow for inhomogeneously broadened resonance lines. Further, the wings of the resonance line can play a more effective role if there is fast diffusion, and the calculated rate will be faster than that obtained neglecting spectral diffusion. Experimental relaxation rates (discussed in $\S 5$ ) are similar to those calculated without spectral diffusion, or even slower, so the fast diffusion model would not improve agreement with experiment. We sum the contributions of the different bands $d E$ to the relaxation rate. As

$$
\tau_{\mathrm{obs}}(E)=\tau_{0}(E)\left\{1+\frac{\tau_{\mathrm{ph}}(E)}{\tau_{0}(E)} \frac{c_{\mathrm{S}}(E)}{c_{\mathrm{L}}(E)}\right\}
$$

using (3) for each band $E$ to $E+d E$, we have

$$
\frac{1}{\tau_{\text {obs }}}=\frac{1}{\tau_{0}} \int d E \frac{g(E)}{1+\left(\tau_{\mathrm{ph}} / \tau_{0}\right)\left\{c_{\mathrm{S}}(E) / c_{\mathrm{L}}(E)\right\}\{g(E) / \gamma(E)\}}
$$

In practice we will be able to neglect the variation of $c_{\mathrm{S}}(E)$ and $c_{\mathrm{L}}(E)$ over the energies of interest. When the second term in the denominator of the integrand is small the integral 
is dominated by the centre of the resonance line, where $g(E)$ is largest. As the bottleneck becomes more severe, this term becomes appreciable and as it is proportional to $g(E)$ the relative importance of the centre of the line is reduced. Correspondingly the wings become more important.

In the case discussed previously the phonon relaxation time was independent of energy, and $g(E)$ was zero outside a band of width $\Gamma$ within which $g(E)$ was constant and equal to $\Gamma^{-1}$. Thus

$$
\tau_{\mathrm{obs}}=\tau_{0}\left(1+\frac{c}{\Gamma}\right)
$$

where, for the direct process,

$$
c=\frac{N \tau_{\text {ph }}}{\rho(\delta) \tau_{0}}\left(\frac{\delta}{2 k T}\right)^{2}
$$

If $g(E)$ is a Lorentzian with full width at half-intensity $\Gamma^{\prime}$

$$
\tau_{\text {obs }}=\tau_{0}\left(1+\frac{2 c}{\pi \Gamma^{\prime}}\right)^{1 / 2}
$$

Comparing this with (10a) shows that when $c$ is small (a slight bottleneck) the observed relaxation time for the Lorentzian of half-width $\Gamma^{\prime}$ is the same as that from (10a) with an 'effective bandwidth' $\Gamma=\pi \Gamma^{\prime}$. For a Gaussian the corresponding 'effective bandwidth' is $\Gamma^{\prime}\{\pi /(2 \ln 2)\}^{1 / 2}$.

As a final example, we choose a Lorentzian $g(E)$ of width $\Gamma_{\mathrm{S}}$ centred on $E=\delta_{\mathrm{S}}$ and a Lorentzian $\gamma(E)$ of width $\Gamma_{\mathrm{p}}$ centred on $E=\delta_{\mathrm{p}}$. Writing $c^{\prime}=2 c / \pi \Gamma_{\mathrm{p}}$ we find

$$
\tau_{\text {obs }}=\tau_{0}\left[1+c^{\prime}\left(\frac{\left(\Gamma_{p}-\Gamma_{S}\right)^{2}+4\left(\delta_{p}-\delta_{S}\right)^{2}}{\Gamma_{p} \Gamma_{S}}\right\}+2 c^{\prime}+\left(c^{\prime}\right)^{2}\right]^{1 / 2} \text {. }
$$

This result is appropriate to the 'one-phonon' processes discussed in the next section. Usually $\delta_{\mathrm{S}}$ and $\delta_{\mathrm{p}}$ vary with an applied magnetic field, so the ratio of $\tau_{\mathrm{obs}}$ to $\tau_{0}$ should be sensitive to this field.

In any real system several different mechanisms may be responsible for spin-lattice relaxation. For example we have assumed that the spins relax independently to the lattice; an alternative is that several spins, coupled by their mutual interaction, change their Zeeman energy simultaneously, with overall energy conservation by the emission or absorption of one phonon. The Temperley effect (Temperley 1939, 1946) and 'cross spin-lattice relaxation' (Bloembergen 1961) fall into the second category. The phonons involved in these different mechanisms will have different energies. If the phonon relaxation time is energy dependent, as in the case leading to $(10 c)$, the relative importance of these mechanisms will be altered when there is a bottleneck. Indeed, mechanisms which are unimportant when the spin-bath relaxation is unrestricted may be dominant when there is a bottleneck.

\section{Phonon relaxation times}

Energy can be transferred from the phonons responsible for relaxation to the bath by several processes, in which the rate-determining process may involve the transfer of energy in space or in frequency. These situations will be described respectively as a spatial bottleneck, for which the bath is usually the liquid helium surrounding the crystal, 
and a spectral bottleneck, for which the lattice vibrations with energies of the order of $k T$ form the bath. Explicit calculations of the phonon relaxation time $\tau_{\mathrm{ph}}$ are given in this section.

\subsection{Spatial bottlenecks}

In a crystal whose dimensions are of order $L$, phonons can reach the bath surrounding the crystal if $v_{c}$, a phonon mean free path, exceeds $L . \tau_{\mathrm{c}}$ is the phonon relaxation time of thermal conductivity, mentioned in $\$ 2$, and $v$ the velocity of sound. If the interface between the crystal and the bath does not reflect phonons, we can calculate $\tau_{\mathrm{ph}}$ by extending results of the theory of black-body radiation (Pippard 1960, pp. 80-1). The energy leaving the crystal per unit area per unit time from the band of phonons resonant with the spins is $\frac{1}{2} v U_{\mathrm{L}}\left(T_{\mathrm{L}}\right)$, where $U_{\mathrm{L}}$ is the energy per unit volume of the lattice system. Correspondingly, the energy entering is $\frac{1}{4} v U_{\mathrm{L}}\left(T_{\mathrm{B}}\right)$. For a crystal of area $A$ and volume $V$

$$
V \frac{d}{d t} U_{\mathrm{L}}\left(T_{\mathrm{L}}\right)=-\frac{1}{4} v A\left\{U_{\mathrm{L}}\left(T_{\mathrm{L}}\right)-U_{\mathrm{L}}\left(T_{\mathrm{B}}\right)\right\}
$$

which can be simplified with the results of $\S 2$ to give

so that

$$
T_{\mathrm{L}}=-\frac{v A}{4 V}\left(T_{\mathrm{L}}-T_{\mathrm{B}}\right)
$$

$$
\tau_{\mathrm{ph}}=4 V / v A
$$

which is $4 L / 3 v$ for a sphere of radius $L$, and $4 L / v$ for a plate of thickness $2 L$. For a spherical crystal, equations $(7 a)$ and $(9 a)$ become

and

$$
\tau_{\text {obs }}=\tau_{0}\left\{1+\frac{4}{3} \frac{L}{v \tau_{\mathrm{c}}(\delta)}\right\}
$$

$$
\tau_{\text {obs }}{ }^{0}=\tau_{0}{ }^{0}\left\{1+\frac{4}{3} \frac{L}{v \tau_{\mathrm{c}}(\Delta)}\right\} .
$$

When the crystal radius is large compared with the phonon mean free path $v \tau_{\mathrm{c}}$ the bottleneck is severe. As (11) is valid when $L \ll v \tau_{c}$ these equations are only quantitatively correct when the bottleneck is slight.

In the limit $L \gg v \tau_{\mathrm{c}}$ it is unreasonable to assume that $T_{\mathrm{I}}$ is homogeneous in space. Eisenstein (1951) obtained an exact solution of the resulting diffusion problem for a sphere of radius $L$ surrounded by a bath. His solution cannot be put into a form corresponding to the last two equations and is usually treated numerically. There is a distribution of relaxation times, rather than a single one. Averaging these over the crystal, and retaining only the lowest order corrections to the unrestricted relaxation time, one can show

$$
\tau_{\mathrm{ph}}=\frac{1}{5} \frac{L}{v} \frac{L}{v \tau_{\mathrm{c}}} \frac{k T}{\delta} .
$$

This is temperature independent, as $\tau_{c}$ is proportional to $T$. Comparison of (11) with the approximate result (12) confirms that corrections for the scatter of phonons within the crystal are necessary when $L \geqslant v \tau_{c}$.

The bath need not be the liquid helium surrounding the crystal, but may be cracks or impurities which transfer the energy to some heat sink so rapidly that the rate-determining process is the transfer of energy in space. In these cases $L$ is related to the mean separation of these defects and is not one of the external dimensions of the crystal. 


\subsection{Spectral bottlenecks}

The phonon relaxation times for spectral bottlenecks depend on the detailed nature of the specimen considered-there are no general results like (11) which depend only on the velocity of sound and the geometry of the specimen.

4.2.1. Anharmonic and similar processes. Van Vleck (1941 b) estimated the energy transfer from the phonon modes resonant with the spins to the thermal modes, with energy of about $k T$, by the anharmonic part of the crystal Hamiltonian. The main process is one in which a thermal phonon and one resonant with the spins unite to form another thermal phonon. This process is about two orders of magnitude more effective for the longitudinal modes than for the transverse modes largely responsible for spinlattice relaxation. Van Vleck shows

$$
\tau_{\mathrm{ph}} \sim \delta^{-1} T^{-4}
$$

for $\mathrm{MgO}$ and $\delta=0.3 \mathrm{~cm}^{-1}, \tau_{\mathrm{ph}}$ is $50 \mu \mathrm{sec}$ at $4.2^{\circ} \mathrm{K}$, falling to $0.08 \mu \mathrm{sec}$ at $21^{\circ} \mathrm{K}$.

In addition to this process, there is one which is closely analogous but in which the anharmonic interaction is replaced by the spin-lattice interaction. This interaction contains terms of linear, quadratic, cubic and higher order in the normal coordinates $Q_{\alpha}$ of the environment of each magnetic ion. These can cause transitions in the phonon system similar to those caused by the anharmonic interaction. Such transitions come, for example, from first-order perturbation by the terms cubic in the $Q_{\alpha}$, or from third-order perturbation by the terms linear in the $Q_{\alpha}$. The calculation of $\tau_{\mathrm{ph}}$ is carried through in close analogy to that of Van Vleck. Several types of contribution result, the most important for the direct process corresponding to combinations of direct and Raman processes, with no net spin flip. For $\mathrm{MgO}: \mathrm{Co}^{2+}$ the terms linear in the $Q_{\alpha}$ are the most important, but this mechanism is less effective than Van Vleck's.

4.2.2. Phonon relaxation via impurities. The situation under consideration is shown in figure 3. The two main requirements of an impurity are a strong interaction with the phonon modes resonant with the spin system studied and a strong interaction with the

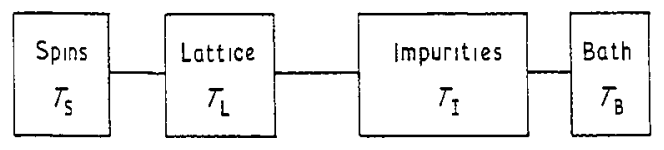

Figure 3.

thermal modes at liquid helium and hydrogen temperatures. It will be assumed that $T_{\mathrm{I}}=T_{\mathrm{B}}$, for one would expect the impurity to be in much better contact with the bath of thermal modes than with the small band of phonons which interact with the spins $S$. The problem reduces to a calculation of the rate at which the phonons resonant with $S$ recover by their interaction with impurities I which are essentially in equilibrium with the bath. For example, if the phonons are nearly resonant with an energy splitting of $I$, they may be absorbed or emitted directly ('single-phonon processes'). Alternatively, the resonant phonon energy may differ from an energy splitting of I by about $k T$, so a phonon resonant with $S$ and a thermal phonon can be absorbed or emitted together ('two-phonon processes'). Higher order processes, involving two or more thermal phonons and one phonon resonant with $S$ (e.g. the anharmonic processes mentioned earlier) prove less important at the low temperatures of interest. 
(i) One-phonon processes. The term in the spin-lattice interaction of the impurity I, linear in the normal coordinates $Q_{\alpha}$, causes transitions in which I absorbs or emits a single phonon. We consider three cases:

(i) The ground state of I consists of two states, $|1\rangle$ and $|2\rangle$, whose energy separation $E_{2}-E_{1}$ is close to the energy $\delta$ of the phonons resonant with S; Raman or Orbach processes maintain $T_{\mathrm{I}}$ close to $T_{\mathrm{B}}$.

(ii) The ground state of $I$ has several energy level separations nearly equal to $\delta$; $T_{\mathrm{I}}$ may be kept close to $T_{\mathrm{B}}$ by Raman or Orbach processes, or by direct process transitions between states separated by energies which are approximately multiples of $\delta$.

(iii) An excited state of $I$ has an energy splitting close to $\delta$, and the ground state has no such splitting.

The temperature dependence of the transition probabilities comes from both the matrix elements of the $Q_{\alpha}$ and the relative populations of the states of I. Writing the temperature-dependent factors explicitly, the rate equation for the number of phonons resonant with the spins $S$ in case (i) is

$$
\frac{d}{d t}\{p(\delta) \Gamma n(\delta)\}=-A(\delta) N_{\mathrm{I}}(1) n(\delta)+A(\delta) N_{\mathrm{I}}(2)\{n(\delta)+1\} .
$$

This is zero when $T_{\mathrm{I}}=T_{\mathrm{B}}$, i.e. when $n(\delta)=n_{0}(\delta)$. Thus

$$
\dot{T}_{\mathrm{L}}=-\frac{A(\delta)\left\{N_{\mathrm{I}}(1)-N_{\mathrm{I}}(2)\right\}}{\rho(\delta) \Gamma}\left(T_{\mathrm{L}}-T_{\mathrm{B}}\right)
$$

The phonon relaxation time is

$$
\tau_{\mathrm{ph}}=\frac{\rho(\delta) \Gamma}{A(\delta)\left\{N_{\mathrm{I}}(1)-N_{\mathrm{I}}(2)\right\}}=\frac{\rho(\delta) \Gamma}{A(\delta)\left\{N_{\mathrm{I}}(1)+N_{\mathrm{I}}(2)\right\}} \frac{2 k T}{\delta}
$$

which is linear in $T$. It can be seen from (7) that both the observed spin-lattice relaxation time of spins $S$ for the direct process and the corresponding unrestricted time are proportional to $T^{-1}$, so in this case the bottleneck cannot be detected by its temperature dependence alone. $\tau_{p h}$, and therefore the observed relaxation time, decreases with increasing impurity concentration. This situation is reminiscent of spin relaxation by cross relaxation to a fast relaxing impurity, a process which may be important in circumstances similar to those for the phonon mechanism just described. The frequency dependence of $\tau_{\mathrm{ph}}(E)$ comes mainly from $A(E)$, which is proportional to the spin-phonon interaction line shape of I. If this is Lorentzian the observed relaxation time is given by (10c), and the observed relaxation time should vary with the applied magnetic field. $A(E)$ is related to the direct process relaxation time, $\tau_{\mathrm{I}}(E)$ for transitions between the states $|1\rangle$ and $|2\rangle$ of $I$ by

$$
A(E)_{\tau_{\mathrm{I}}}(E)=E / 2 k T .
$$

The phonon relaxation time can then be rewritten

$$
\tau_{\mathrm{ph}}(E)=\frac{\rho(E) \tau_{\mathrm{I}}}{\left\{N_{\mathrm{I}}(1)+N_{\mathrm{I}}(2)\right\} g_{\mathrm{I}}(E)}\left(\frac{2 k T}{E}\right)^{2}
$$

where $g_{\mathrm{I}}(E)$ is the acoustic resonance line shape of $\mathrm{I}$.

The phonon relaxation time for case (ii) is also proportional to $T$. This can be shown by explicit calculation, or by noting that, in both (i) and (ii), this dependence comes from 
the population differences of states separated by energies small compared with $k T$ :

$$
T_{\mathrm{L}}=\left(T_{\mathrm{L}}-T_{\mathrm{B}}\right) \sum_{j=1}^{l-1}\left\{N_{\mathrm{I}}(j+1)-N_{\mathrm{I}}(j)\right\} M_{j}
$$

The population differences are linear in $T ; l$ is the number of energy levels of $\mathrm{I}$, and $\mathrm{M}_{j}$ is a temperature-independent factor which describes the variation of the matrix elements involved with $j$.

Equation (13) also holds for case (iii), when $|1\rangle$ and $|2\rangle$ refer to excited states of $I$. The temperature dependence of $\mathrm{N}_{\mathrm{I}}(1)+\mathrm{N}_{\mathrm{I}}(2)$ will cause $\tau_{\mathrm{ph}}$ to decrease roughly exponentially with temperature.

(ii) Two-phonon processes. If the ground state of I consists of two energy levels $|1\rangle$ and $|2\rangle$ whose energy separation $E_{2}-E_{1} \equiv \epsilon$ is of the order of thermal energies, $T_{I}$ will be kept close to $T_{\mathrm{B}}$ by the absorption and emission by I of phonons whose energy is close to $\epsilon$. The two processes in which phonons of energy $\delta$, resonant with $S$, are absorbed are $(A)$ in which the impurity I makes a transition from $|1\rangle$ to $|2\rangle$, and one phonon of energy $\delta$ and one of energy $\epsilon-\delta$ are absorbed, and $(B)$ where $I$ makes a transition from $|2\rangle$ to $|1\rangle$, absorbing a phonon of energy $\delta$ and emitting one of energy $\epsilon+\delta$. There will be corresponding processes in which a phonon of energy $\delta$ is emitted.

The temperature dependence of the phonon relaxation time can be found by computing the net rate of loss of phonons from the band with energy $\delta$ by both (A) and (B). We define $\epsilon_{ \pm}=\epsilon \pm \delta, \theta=\epsilon / k T_{\mathrm{B}}$ and $\phi=\delta / k T_{\mathrm{B}}$. $\phi$ is small, but $\theta$ may be of the order of unity and is in any case much larger than $\phi$. Again we can write down the time dependence of $T_{\mathrm{L}}$, for

$$
\begin{aligned}
\rho(\delta) \Gamma \frac{d}{d t} n(\delta)= & A_{\mathrm{A}}\left[-n(\delta) n\left(\epsilon_{-}\right)+\mathrm{e}^{-\theta}\{n(\delta)+1\}\left\{n\left(\epsilon_{-}\right)+1\right\}\right] \\
& +A_{\mathrm{B}}\left[-n(\delta)\left\{n\left(\epsilon_{+}\right)+1\right\} \mathrm{e}^{-\theta}+\{n(\delta)+1\} n\left(\epsilon_{+}\right)\right]
\end{aligned}
$$

which may be simplified to yield

$$
\dot{T}_{\mathrm{L}}=-\frac{A_{\mathrm{A}}+A_{\mathrm{B}}}{\rho(\delta) \Gamma} \frac{\theta}{\mathrm{e}^{\theta}-1}-\left(T_{\mathrm{L}}-T_{\mathrm{B}}\right)
$$

$A_{\mathrm{A}}$ and $A_{\mathrm{B}}$ are temperature independent by definition. The phonon relaxation time is

$$
\tau_{\mathrm{ph}}=-\frac{\rho(\delta) \Gamma}{A_{\mathrm{A}}+A_{\mathrm{B}}} \frac{\epsilon}{\delta}\left(\frac{\mathrm{e}^{\theta}-1}{\theta}\right)
$$

which is temperature independent when $\theta \equiv \epsilon / k T$ is small. For temperatures such that this is so the relaxation time observed for a strongly bottlenecked direct process should vary as $T^{-2}$, increasing faster with decreasing temperature below $T \sim \epsilon \mid k$ when $\theta$ is of order unity. An explicit calculation of $A_{\mathrm{A}}$ and $A_{\mathrm{B}}$ uses the same methods as the calculation of spin-lattice relaxation times. If we take the terms of the spin-lattice interaction which are linear in the normal coordinates $Q_{\alpha}$ of the environment of $\mathrm{I}$,

$$
\frac{1}{\tau_{p h}}=\frac{8}{27 \pi} N_{\mathrm{I}} \frac{\epsilon^{2}}{\hbar^{4} \rho^{2} v^{7}}\left|\sum_{\substack{i=1,2 \\ \alpha, \beta}}\left\langle 2\left|W_{\alpha}\right| i\right\rangle\left\langle i\left|W_{\beta}\right| 1\right\rangle\right|^{2} \frac{\theta}{e^{\theta}-1} .
$$

In this result $W_{\alpha}$ is the coefficient of $Q_{\alpha} / R$ in the spin-lattice interaction, $R$ is the distance from $I$ to one of its ligands and $\rho$ the density of the crystal. The energies of excited states $|i\rangle$, other than $|2\rangle$, above the ground state $|1\rangle$ have been assumed 
appreciably larger than $\epsilon$. The spread in $\epsilon$ has been neglected, although it is important that the impurities interact with a sufficient band of phonons to stay close to the bath temperature. $\tau_{\mathrm{ph}}$ does not depend on $\delta$, so in a strong bottleneck the observed relaxation time should be independent of applied magnetic fields. This is a complete contrast to the single-phonon process which, as discussed in $\$ 3$, should be strongly field dependent.

As an example of a two-phonon process, K. W. H. Stevens (unpublished) has suggested that the lowest states of $\mathrm{Ti}^{3+}$ in $\mathrm{MgO}$ are split by about $6^{\circ}$, and that processes of the type described are responsible for a high observed attenuation of ultrasonics. Van Vleck (1940) has given the matrix for the $\mathrm{Ti}^{3+}$ spin-lattice interaction in terms of two coefficients $a$ and $b$. These are analogous to our $W_{\alpha} / R$, the coefficient of $Q_{\alpha}$ in the spin-lattice interaction. Typical values are $a R \sim b R \sim 10^{4} \mathrm{~cm}^{-1}$ for iron group elements, although these must be reduced by a factor $\frac{1}{3}$ for $\mathrm{Ti}^{3+}$ (Van Vleck 1960). If we assume our states $|1\rangle$ and $|2\rangle$ are the $\left|E_{a}\right\rangle$ and $\left|E_{b}\right\rangle$ of Van Vleck then

$$
\tau_{\mathrm{ph}} \sim 1.6 \times 10^{-6} n_{\mathrm{I}}^{-1} \mathrm{sec}
$$

where $n_{\mathrm{I}}$ is the number of $\mathrm{Ti}^{3+}$ ions per $10^{6} \mathrm{Mg}^{2+}$ sites. $\mathrm{Cr}^{2+}$ and $\mathrm{V}^{3+}$ in some host lattices provide further examples of impurities which have energy levels a few degrees above their ground state (Low 1960).

\section{Discussion}

We may summarize the various phonon relaxation mechanisms as follows:

(1) Spatial bottlenecks. Here the rate-determining process is the transfer of energy in space. (a) The phonons pass through the crystal surface to the crystal environment. When the phonon mean free path $v \tau_{0}$ is much larger than the crystal 'radius' $L,(11)$ gives

$$
\tau_{\mathrm{ph}}{ }^{-1} \sim L^{-1} \text {. }
$$

When $L$ is much larger than $v \tau_{0}$ Eisenstein's (1951) results must be used. (b) The phonons travel to defects which transfer their energy to the lattice in a time which is short compared with the travel time. The travel time will depend on the concentration $f_{1}$ of the defects, whose mean separation varies as $f_{1}^{-1 / 3}$; thus one expects that

$$
\tau_{\text {ph }} \sim f_{\mathrm{r}}-1 / 3 .
$$

(2) Spectral bottlenecks. Here the transfer of energy in frequency is the rate-determining process. (a) In the single-phonon process, for which $\delta$ is close to an energy splitting of the impurity,

$$
\tau_{\mathrm{ph}}^{-1} \sim f_{1} T^{-1} \text {. }
$$

(b) In the two-phonon process, for which $\delta$ differs from an energy splitting of the impurity $\in$ by about $k T$,

$$
\tau_{\mathrm{ph}}^{-1} \sim f_{\mathrm{s}}
$$

(c) For the anharmonic processes

$$
\tau_{\mathrm{ph}}^{-1} \sim \delta T^{4} .
$$

$f_{\mathrm{I}}$ is the concentration of the impurity responsible for phonon relaxation.

The phonon relaxation time is important in relating the observed relaxation time to the unrestricted spin-lattice relaxation time. For the direct process in a strong bottleneck $\tau_{\mathrm{obs}}{ }^{-1} \sim \tau_{\mathrm{ph}}{ }^{-1} T^{2} \Gamma / f$ where $\Gamma$ is the bandwidth of phonons resonant with spins and $f$ the concentration of $S$; we have omitted the energy dependence of $\tau_{\mathrm{ph}}$ and the effects of line shape described in $\S 3$. Thus $\tau_{p h}$ must be independent of temperature for the $T^{-2}$ 
dependence of $\tau_{o b s}$, usually regarded as a symptom of a bottleneck. This is so in three cases only, the spatial bottlenecks $(1 a)$ and $(1 b)$ and the two-phonon process $(2 b)$. These can be distinguished experimentally, for all give a different dependence on the impurity concentration $f_{\mathrm{r}}$. (1a) alone depends on the dimensions of the crystal, and the $T^{2}$ rule breaks down for ( $2 b)$ below a temperature $\epsilon / k$. The one-phonon process $(2 a)$ and the anharmonic processes $(2 c)$ give a $T$ and a $T^{6}$ dependence for $\tau_{\text {obs }}$, respectively.

There are quite a few experimental results with which (11) and Eisenstein's (1951) results can be compared. When (11) should apply the observed phonon relaxation times obtained (Nash 1961, Scott and Jeffries 1962, Standley and Wright 1962, 1964, J. C. Gill, private communication) usually lie between one and six times those predicted, including the effects of line shape by use of $(10 b)$. Presumably the longer times are partly due to phonon scatter within the crystal, when the assumption $L \ll v \tau_{c}$ is not strictly valid, and partly to the reflection of phonons incident on the crystal surface. The acoustic mismatch between the crystals and the liquid helium suggests almost complete reflection should occur; this would lengthen the observed relaxation times. Mills (1964) suggested that, as all the crystals for which agreement is satisfactory are those with water of crystallization, they may become dehydrated near the surface. This would form a porous structure which may fill with liquid helium to give an essentially non-reflecting layer. If the crystal surface is then dissolved away and the crystal used immediately, we may expect that there will be little dehydration and that the surface may be polished by the treatment. The observed relaxation times should be longer than those predicted assuming no reflection, if Mills' suggestion is correct. This has been observed by Gill, whose experiments give some support to this theory.

Several cases have been found (Van den Broek et al. 1961, Van der Marel et al. 1957) in which the Eisenstein model gives good agreement with experiment, using crystals of dimensions of the order of centimetres.

The two-phonon mechanism may have been observed in experiments on $\mathrm{MgO}$ crystals doped intentionally with $\mathrm{Co}^{2+}$ and unintentionally with other iron group ions (Whittlestone 1964, Ph.D. Thesis, University of Bristol). The phonon relaxation times were of the order of $10^{-7} \mathrm{sec}$, varying from crystal to crystal, and were temperature independent. They did not depend on the crystal size, confirming the estimate from (11) that mechanism (1a) was inadequate. (15) suggests that these results could be explained by about ten $\mathrm{Ti}^{3+}$ ions per $10^{6} \mathrm{Mg}^{2+}$ sites, which is a typical value for impurity concentrations in the crystals used. On the other hand the $T^{2}$ dependence of $\tau_{\text {obs }}{ }^{-1}$ continued below the assumed value of $\epsilon \mid k \sim 6^{\circ}$. The results are, of course, consistent with mechanism (1b). These measurements appear to be the only systematic set for crystals without water of crystallization. The main uncertainty lies in our knowledge of the impurities present in small but important concentrations; whilst it is possible to find explanations consistent with the results, it is not yet possible to make reliable predictions. Both the phonon relaxation mechanisms consistent with the results would be absent in a perfect $\mathrm{MgO}$ crystal containing only single $\mathrm{Co}^{2+}$ impurities.

\section{Acknowledgments}

The author wishes to thank Dr. J. C. Gill and Dr. P. R. Whittlestone for the use of some of their unpublished results, and Dr. A. B. Lidiard for very helpful discussions.

\section{References}

Bloembergen, N., 1961, Advances in Quantum Electronics, Ed. J. R. Singer (New York: Columbia University Press), pp. 384-401.

Brya, W. J., and WAGNER, P. E., 1965, Phys. Rev. Letters, 14, 431-3. 
Casimir, H. B. G., 1939, Physica, 6, 156-62.

EISENSTEIN, J., 1951, Phys. Rev., 84, 548-50.

Faughinan, B. W., and Strandierg, M. W. P., 1961, J. Phys. Chem. Solids, 19, 155-66.

Giel, J. C., and Meredith, D. J., 1965, Phys. Letters, 15, 201-2.

Gorter, C. J., Van Der Marel, L. C., and Bolger, B., 1955, Physica, 21, 103-18.

KIEMENS, P. J., 1958, Solid St. Phys., 7, 1-96.

Loudon, R., 1960, Phys. Rev., 119, 919-21.

Low, W., 1960, Solid St. Phys. (Suppl. 2), 1-212

Mitls, D. L., 1964, Phys. Rev. 133, A876-86.

Nash, F. R., 1961, Phys. Rev. Letters, 7, 59-61.

Orbach, R., 1961, Proc. Roy. Soc. A, 264, 458-84.

— 1962, Phys. Rev. Letters, 8, 393-6.

PIPPARd, A. B., 1960, The Elements of Classical Thermodynamics (London: Cambridge University Press).

Portis, A. M., 1956, Phys. Rev., 104, 584-8.

SCOTt, P. L., and Jefreries, C. D., 1962, Phys Rev., 127, 32-51.

StANDley, K. J., and Wright, J. K., 1962, Phys. Letters, 3, 101-2.

- 1964, Proc. Phys. Soc., 83, 361-8.

Stoneham, A. M., 1965, Phys. Letters, 14, 297-8.

Temperdey, H. N. V., 1939, Proc. Camb. Phil. Soc., 35, 256-64.

- 1946, Proc. Camb. Phil. Soc., 43, 119-26.

Tolman, R. C., 1938, The Principles of Statistical Mechanics (London: Oxford University Press).

Van den Broek, J., and VAN DER Marel, L. C., 1963, Physica, 29, 948-64.

VAN den Broek, K., VAN Der Marel, L. C., and Gorter, C. J., 1961, Physica, 27, 661-80.

Vai der Marel, L. C., VAan Den Broek, J., and Gorter, C. J., 1957, Physica, 23, 361-77.

VAN VleCK, J. H., 1940, Phys. Rev., 57, 426-47.

1941 a, Phys. Rev., 59, 724-9.

1941 b, Phys. Rev., 59, 730-6.

1959, Quantum Electronics, Ed. C. H. Townes (New York: Columbia University Press), pp. 392-409.

1960, Physica, 26, 548-52. 